\title{
Analysis of the nonleptonic two-body decays of the $\Lambda$ hyperon
}

\author{
Mikhail A. Ivanov, ${ }^{1}$ Jürgen G. Körner, ${ }^{2}$ Valery E. Lyubovitskij $\odot,{ }^{3,4,5,6,7}$ and Zhomart Tyulemissov ${ }^{1,8,9}$ \\ ${ }^{1}$ Bogoliubov Laboratory of Theoretical Physics, Joint Institute for Nuclear Research, Dubna, Russia \\ ${ }^{2}$ PRISMA Cluster of Excellence, Institut für Physik, Johannes Gutenberg-Universität, \\ D-55099 Mainz, Germany \\ ${ }^{3}$ Institut für Theoretische Physik, Universität Tübingen, Kepler Center for Astro \\ and Particle Physics, Auf der Morgenstelle 14, D-72076 Tübingen, Germany \\ ${ }^{4}$ Departamento de Física y Centro Científico Tecnológico de Valparaíso-CCTVal, \\ Universidad Técnica Federico Santa María, Casilla 110-V, Valparaíso, Chile \\ ${ }^{5}$ Millennium Institute for Subatomic Physics at the High-Energy Frontier (SAPHIR) of ANID, \\ Fernández Concha 700, Santiago, Chile \\ ${ }^{6}$ Department of Physics, Tomsk State University, 634050 Tomsk, Russia \\ ${ }^{7}$ Tomsk Polytechnic University, 634050 Tomsk, Russia \\ ${ }^{8}$ The Institute of Nuclear Physics, Ministry of Energy of the Republic of Kazakhstan, \\ 050032 Almaty, Kazakhstan \\ ${ }^{9}$ Al-Farabi Kazakh National University, 050040 Almaty, Kazakhstan
}

(Received 20 July 2021; accepted 17 September 2021; published 8 October 2021)

\begin{abstract}
We systematically study two-body nonleptonic decays of light lambda hyperon $\Lambda \rightarrow p \pi^{-}\left(n \pi^{0}\right)$ with account for both short- and long-distance effects. The short-distance effects are induced by five topologies of external and internal weak $W^{ \pm}$exchange, while long-distance effects are saturated by an inclusion of the so-called pole diagrams with intermediate $\frac{1}{2}^{+}$and $\frac{1}{2}^{-}$baryon resonances. The contributions from $\frac{1}{2}^{+}$ resonances are calculated straightforwardly by account for nucleon and $\Sigma$ baryons whereas the contributions from $\frac{1}{2}-$ resonances are calculated by using the well-known soft-pion theorem in the current-algebra approach. It allows one to express the parity-violating $S$-wave amplitude in terms of parityconserving matrix elements. From our previous analysis of heavy baryons we know that short-distance effects induced by internal topologies are not suppressed in comparison with external $W$-exchange diagram and must be included for description of data. Here, in the case of $\Lambda$ decays we found that the contribution of external and internal $W$-exchange diagrams is sizably suppressed, e.g., by one order of magnitude in comparison with data, which are known with quite good accuracy. Pole diagrams play the major role to get consistency with experiment.
\end{abstract}

DOI: 10.1103/PhysRevD.104.074004

\section{INTRODUCTION}

Two-body nonleptonic decays of the $\Lambda$ hyperon $\Lambda \rightarrow$ $p \pi^{-}$and $\Lambda \rightarrow n \pi^{0}$ are the leading modes with branching fractions $63.9 \pm 0.5 \%$ and $35.8 \pm 0.5 \%$ [1], respectively. These processes follow by producing $\Lambda$ in the $K^{-} p$ scattering and are clearly identified after substraction of background effects. The other modes of the $\Lambda$ are suppressed by a few orders of magnitude. Such precise data on the $\Lambda \rightarrow N \pi$ decays give a unique possibility to consider these decays as a laboratory for testing nature of the

Published by the American Physical Society under the terms of the Creative Commons Attribution 4.0 International license. Further distribution of this work must maintain attribution to the author(s) and the published article's title, journal citation, and DOI. Funded by SCOAP ${ }^{3}$. weak decays and a crucial check of existing theoretical approaches modeling exclusive decays of baryons. From a modern theoretical point of view, there are two classes of the Feynman diagrams generating matrix elements of these processes: (1) short-distance (SD) diagrams and (2) longdistance (LD) or pole diagrams. The SD diagrams are classified by five different color-flavor topologies as shown in Fig. 1. We refer to the topologies of Ia and Ib as tree diagrams. They are sometimes also referred to as external (Ia) and internal $W$-emission (Ib) diagrams. The topologies $\mathrm{IIa}$, Ilb, and III are referred to as $W$-exchange diagrams. Note, in literature one can find other notations for $W$-exchange diagrams. For examle, in [2] they are denoted as the exchange (IIa), color-commensurate (IIb), and bow tie (III) diagram. As shown in Fig. 1 the color-flavor factor of the tree diagrams Ia and Ib depends on whether the emitted meson is charged or neutral. For charged emission the color-flavor factor is given by the linear 


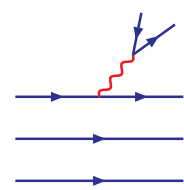

Ia

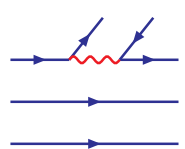

$\mathrm{Ib}$

Tree diagrams

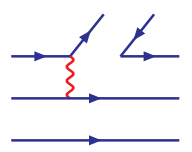

IIa

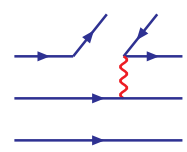

IIb

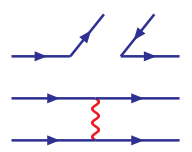

III
W-exchange diagrams

FIG. 1. Flavor-color topologies of nonleptonic weak decays.

combination of Wilson coefficients $\left(C_{2}+\xi C_{1}\right)$, where $\xi=1 / N_{c}$, while for neutral emission the color-flavor factor reads $\left(C_{1}+\xi C_{2}\right)$. We use the large- $N_{c}$ limit for the color-flavor factors with $\xi=0$. For the $W$-exchange diagrams the color-flavor factor is given by $\left(C_{2}-C_{1}\right)$. The Wilson coefficients $C_{2}=1.361$ (leading order) and $C_{1}=$ -0.625 (subleading order) are taken at the scale $\mu=$ $1 \mathrm{GeV}$, on $\Lambda \frac{(4)}{\mathrm{MS}}=435 \mathrm{MeV}$ in the Naive Dimensional Regularization (NDR) scheme from Ref. [3]. Note, the contribution of the QCD penguin diagrams to the nonleptonic decays of the $\Lambda^{0}$ hyperon is strongly suppressed in comparison with the contribution of the current-current operators due to a suppression of the corresponding Wilson coefficients.

In Refs. [4-14] we have performed comprehensive analysis of the nonleptonic decays of the single and double heavy baryons including topologies with external and internal $W$ exchange. In particular, we have shown that the internal topologies are not always suppressed and must be systematically included in theoretical analysis. Here we turn to study of nonleptonic decays of the light baryons, which up to now were more precisely determined from the data and could give an opportunity for an additional check of the theoretical approaches.

Since the end of the 1960s two-body nonleptonic decays of the $\Lambda$ hyperon have been studied in the literature using different approaches. One of the first attempts to the $\Lambda$ and other hyperons has been done by using effective weak Hamiltonians and methods of current algebra, dispersion relations, and vector dominance [15-24]. In Ref. [25] nonleptonic decays of hyperons were analyzed using three types of nonrelativistic bound-state models of baryons: a quark model, a three-triplet model, and a quartet model. In the 1970s significant progress was achieved in studying nonleptonic decays of hadrons (e.g., kaons and hyperons). It was shown (for review, see Ref. [26]) that short-distance strong effects playing important role in weak interactions of hadrons can be systematically included in QCD, which lead to modification of effective weak Hamiltonians by dressing their couplings and arising of new operators beyond a $V-A$ structure. In this vein, effective weak Hamiltonians (for review see, e.g., Ref. [3]) are constructed which further their use in evaluation of matrix elements to predict nonleptonic decay properties of hadrons. First, systematic application of these ideas to hyperons and also to kaons can be done in Ref. [26]. In particular, in Ref. [26] the most general form of the effective weak Hamiltonian dressed by strong short-distance effects and containing four-quark operators with specific quantum numbers of isospin and unitary spin has been derived. Dressing of the couplings in operators due to gluon corrections has been taken into account by solving corresponding renormalization group equations. Finally, matrix elements of nonleptonic transitions have been evaluated using the valence quark approximation. This work [26] served as the basis for modern theory of weak decays of hadrons. The effective weak Hamiltonian is a universal ingredient in calculation of matrix elements of physical processes and all model ambiguities are encoded in a way of projection of the Hamiltonian between respective hadronic states or to evaluation of matrix elements. Note that significant progress in studying nonleptonic decays of light baryons has been made in the framework of the effective field theories [27-36]. In particular, different types of effective theories [chiral perturbation theory (ChPT), heavy baryon ChPT (HBChPT), and large- $N_{c}$ QCD] with implementation of chiral and $1 / N_{c}$ corrections, isospin, and $\mathrm{SU}(3)$ breaking correction for study of hyperon decays have been developed. It was found that an extension of ChPT beyond leading order is extremely important and one-loop/chiral corrections became significant for the $p$-wave hyperon amplitudes. Recently, an updated analysis of light baryon nonleptonic decays in ChPT has been performed in Ref. [35] with taking into account large- $N_{c}$ expansion and $\mathrm{SU}(3)$ symmetry-breaking effects. In Ref. [36] the contributions to the $C P$-violating asymmetries induced by the chromomagnetic-penguin operators have been studied. In Ref. [37] weak and radiative decays of hyperons have been considered in a pole model. In Ref. [38] nonleptonic decays of hyperons were analyzed in a constituent quark model. Skyrme soliton model was applied for a description of weak nonleptonic decays of hyperons in Refs. [39,40]. The MIT bag model to nonleptonic decays of baryons has been applied in Ref. [41]. Phenomenological analysis of nonleptonic decays of hyperons-based chiral Lagrangian model was done in Ref. [42]. Sum rules for the nonleptonic hyperon decays have been derived in Refs. [43-46]. In Ref. [47] nonleptonic decays have been considered in the nonrelativistic potential model. In Ref. [48] nonleptonic decays of hyperons have been analyzed in quark-diquark model. SU(4)-flavor chiral soliton model was applied to a description of hyperon nonleptonic decays in Ref. [49]; the 
two-body nonleptonic decays of light baryon octet and decuplet have been studied using a combination of the topological diagram approach and the $\mathrm{SU}(3)$ irreducible representation approach, i.e., the transition amplitudes are derived as linear combinations of the basic amplitudes classified accordingly to irreducible representations of the unitary flavor group and constraints due to isospin symmetry we also implemented. The main conclusion of Ref. [50] is that $W$-exchange diagrams give large and even dominant contributions to the decay rates. Note that in these papers, a nontrivial nonperturbative dynamics of quarks in hadrons and in the intermediated stage in propagating between individual hadrons was modeled by the dipolelike form factors [51] or even ignored as in Ref. [50]. From our analysis of nonleptonic decays we know that it is a sharp approximation, which not always matches data. In particular, one can describe data up to order of magnitude, but not precise. A modeling of the internal nonperturbative dynamics of hadronic constituents is needed in study of exclusive decays of baryons. In Ref. [52] two-body nonleptonic decays of the $\Lambda$ have been considered in an effective quark model with chiral $U(3) \times U(3)$ symmetry. Partial decay rates and angular distributions have been calculated. Based on $\mathrm{SU}(2)$, spin $\mathrm{SU}(3)$-flavor symmetries and vector dominance joined description of weak radiative and nonleptonic decays of light hyperons has been done in Ref. [53]. In Ref. [54] factorizing and pole contributions to the nonleptonic decays of light hyperons have been evaluated taking into account the instanton contributions in the framework of the Random Instanton Liquid Model. It was concluded that roughly $70 \%$ of the amplitudes come from instanton-induced interactions (responsible for the spontaneous breaking of chiral symmetry), $10 \%$ from hard gluon-exchange corrections, and the remaining $20 \%$ were due to confinement effect. A very weak dependence on a choice of current quark mass was noticed. In Ref. [55] a potential model explicitly incorporating quark-quark correlations was applied to nonleptonic decays of light hyperons. In Ref. [56] an algebraic approach based on mixing hyperons was used for calculation of their nonleptonic decay rates.

The main objective of the present paper is to investigate a role of $W$ - exchange diagrams in strange hyperon physics. It is well known that the factorizable diagrams dominated by SD effects [41,44,57], in principle, describe data well. However, it does not mean that $W$-exchange and LD diagrams vanish. They could nontrivially interplay with SD factorizable contributions. Nonfactorizable diagrams in baryon nonleptonic decays play an important role. The analysis of nonleptonic baryon decays is complicated by the necessity of having to include nonfactorizing contributions. One thus has to go beyond the factorization approximation which had proved quite useful in the analysis of the exclusive nonleptonic decays of heavy mesons. There have been some theoretical attempts to analyze nonleptonic heavy baryon decays using factorizing contributions alone, the argument being that $W$-exchange contributions can be neglected in analogy to the powersuppressed $W$-exchange contributions in the inclusive nonleptonic decays of heavy baryons. One might even be tempted to drop the nonfactorizing contributions on account of the fact that they are superficially proportional to $1 / N_{c}$. However, since $N_{c}$ baryons contain $N_{c}$ quarks an extra combinatorial factor proportional to $N_{c}$ appears in the amplitudes which cancels the explicit diagrammatic $1 / N_{c}$ factor. Another argument supporting importance of study of $W$-exchange diagrams is that there are the modes which are nonsuppressed and proceed only via these diagrams. (see detailed discussion in Refs. [13,58]). In Ref. [13] we showed that the total contribution of the nonfactorizing diagrams can amount up to $60 \%$ of the factorizing contribution for heavy-to-light baryon transitions and up to $30 \%$ for $b \rightarrow c$ baryon transitions. Recently we improved our formalism for study of the nonfactorizable diagrams in the nonleptonic decays of heavy baryons. We have made an $a b$ initio three-loop quark model calculation of the $W$-exchange contribution to the nonleptonic two-body decays of the doubly charmed baryons $\Xi_{c c}^{++}$and $\Omega_{c c}^{+}$. The $W$-exchange contributions appear in addition to the factorizable tree graph contributions and are not suppressed in general. We made use of the covariant confined quark model previously developed by us to calculate the tree graph as well as the $W$-exchange contribution. We calculated helicity amplitudes and quantitatively compare the tree graph and $W$-exchange contributions. Finally, we compared the calculated decay widths with those from other theoretical approaches when they are available. We found a substantial contribution of $W$-exchange graphs to the modes with final baryon containing spin-0 light diquarks. The suppression of the $W$ graphs for the modes with final baryons containing spin-1 light diquarks is explained by the consistency of our framework with the Körner, Pati, Woo (KPW) theorem [59,60], which states that the contraction of the flavor-antisymmetric currentcurrent operator with a flavor-symmetric final-state configuration is zero in the SU(3) limit. In Ref. [6] we made unified analysis of semileptonic and nonleptonic two-body decays of the double-charm baryon ground states $\Xi_{c c}^{++}, \Xi_{c c}^{+}$, and $\Omega_{c c}^{+}$. We identified those nonleptonic decay channels in which the decay proceeds solely via the factorizing contribution precluding a contamination from $W$ exchange. We use the covariant confined quark model previously developed by us to calculate the various helicity amplitudes which describe the dynamics of the $1 / 2^{+} \rightarrow 1 / 2^{+}$and $1 / 2^{+} \rightarrow 3 / 2^{+}$transitions induced by the Cabibbo favored effective $(c \rightarrow s)$ and $(d \rightarrow u)$ currents. We then proceed to calculate the rates of the decays as well as polarization effects and angular decay distributions of the prominent decay chains resulting from the nonleptonic decays of the double heavy charm baryon parent states. Taking into 
account above arguments we conclude that taking into account of $W$-exchange graphs and development of theoretical methods for their evaluation is quite an important task.

The paper is structured as follows. In Sec. II we give the basic ingredients of our framework which includes the effective Hamiltonian of the weak interactions and the description of quark structure of hadrons in the covariant confined quark model. Sec. III is devoted to calculation of the matrix elements of the decays $\Lambda \rightarrow p+\pi^{-}$and $\Lambda \rightarrow n+\pi^{0}$. We discuss in detail the classification of the Feynman diagrams appearing in these decays and give the analytical expressions for matrix elements and helicity amplitudes. In Sec. IV we present numerical results for comprehensive analysis of nonleptonic decays of $\Lambda$ hyperon. Finally, in Sec. V we make conclusions and summarize the main results obtained in this paper.

\section{EFFECTIVE HAMILTONIAN AND DIAGRAMS}

We concentrate our discussion on two nonleptonic decay modes of $\Lambda$ - hyperon: $\Lambda^{0} \rightarrow p+\pi^{-}$and $\Lambda^{0} \rightarrow n+\pi^{0}$. They proceed due to weak interactions of quarks which are described by the effective Hamiltonian:

$$
\begin{aligned}
\mathcal{H}_{\mathrm{eff}}= & -\frac{G_{F}}{\sqrt{2}} V_{\mathrm{CKM}}\left\{C_{2}\left(\bar{u}_{a} O_{L} s_{a}\right)\left(\bar{d}_{b} O_{L} u_{b}\right)\right. \\
& \left.+C_{1}\left(\bar{u}_{a} O_{L} s_{b}\right)\left(\bar{d}_{b} O_{L} u_{a}\right)\right\}
\end{aligned}
$$

where $V_{\mathrm{CKM}}=V_{\mathrm{ud}} V_{\mathrm{us}}^{*}$ is the product of the CabibboKobayashi-Maskawa matrix elements; the matrix $O_{L}^{\mu}=$ $\gamma^{\mu}\left(I-\gamma_{5}\right)$ is the weak matrix with the left chirality. The summation over $\mu$ is implied in Eq. (1). The Wilson coefficients $C_{2}$ (leading order) and $C_{1}$ (subleading order) are taken at the scale $\mu=1 \mathrm{GeV}$, on $\Lambda_{\overline{\mathrm{MS}}}^{(4)}=435 \mathrm{MeV}$ in the NDR scheme from Ref. [3] (see Table XVIII): $C_{1}=-0.625, C_{2}=1.361$.

We should stress that the contribution of the QCD penguin diagrams to the nonleptonic decays of the $\Lambda^{0}$ hyperon is strongly suppressed in comparison with the contribution of the current-current operators because the Wilson coefficients $C_{3}-C_{6}$ corresponding to the penguin operators are suppressed at least by two orders of magnitude in comparison with the Wilson coefficients $C_{1}$ and $C_{2}$. We present a comparison of the Wilson coefficients $C_{1}, C_{2}$ for the current-current operators and $C_{3}-C_{6}$ for the QCD penguins in Table I.

We will take into account both the SD diagrams and LD diagrams contributions. In general, the SD diagrams have

TABLE I. Wilson coefficients.

\begin{tabular}{lccccc}
\hline \hline$C_{1}$ & $C_{2}$ & $C_{3}$ & $C_{4}$ & $C_{5}$ & $C_{6}$ \\
\hline-0.2632 & 1.0111 & -0.0055 & -0.0806 & 0.0004 & 0.0009 \\
\hline \hline
\end{tabular}
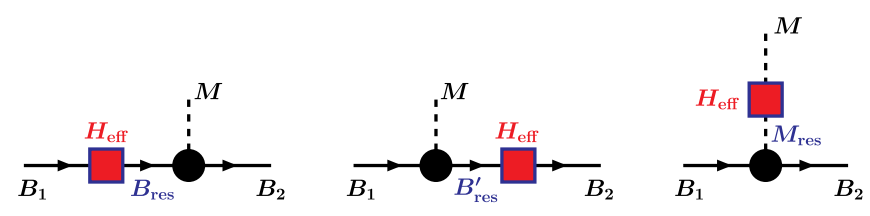

FIG. 2. The pole diagrams which effectively account for the long-distance contributions.

five different topologies generated by the $W$-exchange between two quarks as shown in Fig. 1. Note the $W$-exchange diagram III contributes to the $S$-wave amplitude, because the light diquark loop with weak nonleptonic vertex contains the Dirac structure proportional fourdimensional Levi-Cevita tensor $\epsilon^{\alpha, \beta, \rho, \sigma}$, which produces $S$-wave amplitude due to contracting with to the fermion line containing two quark propagators and $\gamma^{5}$ Dirac matrix (due to pion coupling to $u / d$ quarks). One should stress that the contribution of the diagram III to the $S$-wave amplitude could vanish in some limiting cases. For example, in Refs. [13,58] it occurs when a static approximation for baryon wave functions or light quark propagators is used. In the present paper we go beyond static approximation.

In addition to the SD diagrams we calculate the pole diagrams as shown in Fig. 2 by account for the lowest-lying resonances with spin $1 / 2$ and 0 .

In Table II we display the quantum numbers, mass values, and interpolating currents of baryons needed in this paper. Note that the forms of interpolating currents are not unique. The different form may be transformed to each other by using the Fierz transformations. We do not display the overall flavor factors because they will be recovered due to $T$-product operation of the quark fields.

We are going to calculate the matrix elements of the above-mentioned nonleptonic decays in the framework of the covariant confined quark model (CCQM) developed in our previous papers. The starting point is the Lagrangian describing couplings of the baryon field with its interpolating quark current:

$$
\mathcal{L}(x)=g_{B}(\bar{B}(x) \cdot J(x)+\bar{J}(x) \cdot B(x)),
$$

where $\bar{J}=J^{\dagger} \gamma^{0}$ is the Dirac conjugate current. The coupling constant $g_{B}$ is determined from the normalization condition called compositeness condition.

The nonlocal version of the interpolating currents shown in Table II reads

$$
\begin{aligned}
J_{B}(x)= & \int d x_{1} \int d x_{2} \int d x_{3} F_{B}\left(x ; x_{1}, x_{2}, x_{3}\right) \varepsilon_{a b c} \\
& \times \Gamma_{1} q_{1}^{a}\left(x_{1}\right)\left(q_{2}^{b}\left(x_{2}\right) C \Gamma_{2} q_{3}^{c}\left(x_{3}\right)\right), \\
F_{B}= & \delta^{(4)}\left(x-\sum_{i=1}^{3} w_{i} x_{i}\right) \Phi_{B}\left(\sum_{i<j}\left(x_{i}-x_{j}\right)^{2}\right),
\end{aligned}
$$


TABLE II. Quantum numbers and interpolating currents of baryons.

\begin{tabular}{llrl}
\hline \hline Baryon & $J^{P}$ & Interpolating current & Mass (MeV) \\
\hline$\Lambda^{0}$ & $\frac{1}{2}^{+}$ & $\varepsilon_{a b c} s^{a}\left(u^{b} C \gamma_{5} d^{c}\right)$ & $1115.683 \pm 0.006$ \\
$\Sigma^{+}$ & $\frac{1}{2}^{+}$ & $\varepsilon_{a b c} \gamma_{\mu} \gamma_{5} s^{a}\left(u^{b} C \gamma^{\mu} u^{c}\right)$ & $1189.37 \pm 0.07$ \\
$\Sigma^{0}$ & $\frac{1}{2}^{+}$ & $\varepsilon_{a b c} \gamma_{\mu} \gamma_{5} s^{a}\left(u^{b} C \gamma^{\mu} d^{c}\right)$ & $1192.642 \pm 0.024$ \\
$\Delta^{0}$ & $3^{+}$ & $\varepsilon_{a b c}\left\{u^{a}\left(d^{b} C \gamma^{\mu} d^{c}\right)-\frac{i}{2} \gamma_{\nu} u^{a}\left(d^{b} C \sigma^{\mu \nu} d^{c}\right)\right\}$ & $1231.3 \pm 0.6$ \\
$\Sigma^{*+}$ & $3^{+}$ & $\varepsilon_{a b c}\left\{s^{a}\left(u^{b} C \gamma^{\mu} u^{c}\right)-\frac{i}{2} \gamma_{\nu} s^{a}\left(u^{b} C \sigma^{\mu \nu} u^{c}\right)\right\}$ & $1382.80 \pm 0.35$ \\
$\Sigma^{* 0}$ & $\frac{3}{2}^{+}$ & $\varepsilon_{a b c}\left\{s^{a}\left(u^{b} C \gamma^{\mu} d^{c}\right)-\frac{i}{2} \gamma_{\nu} s^{a}\left(u^{b} C \sigma^{\mu \nu} d^{c}\right)\right\}$ & $1383.7 \pm 1.0$ \\
$p$ & $\frac{1}{2}^{+}$ & $\varepsilon_{a b c}\left\{(1-x) \gamma_{\mu} \gamma_{5} d^{a}\left(u^{b} C \gamma^{\mu} u^{c}\right)-\frac{x}{2} \sigma_{\mu \nu} \gamma_{5} d^{a}\left(u^{b} C \sigma^{\mu \nu} u^{c}\right)\right\}$ & $938.2720813 \pm 0.0000058$ \\
$n$ & $\frac{1}{2}^{+}$ & $\varepsilon_{a b c}\left\{(1-x) \gamma_{\mu} \gamma_{5} u^{a}\left(d^{b} C \gamma^{\mu} d^{c}\right)-\frac{x}{2} \sigma_{\mu \nu} \gamma_{5} u^{a}\left(d^{b} C \sigma^{\mu \nu} d^{c}\right)\right\}$ & $939.5654133 \pm 0.0000058$ \\
\hline \hline
\end{tabular}

where $w_{i}=m_{i} /\left(\sum_{j=1}^{3} m_{j}\right)$ and $m_{i}$ is the quark mass at the space-time point $x_{i}$, and $\Gamma_{1}, \Gamma_{2}$ are the Dirac strings of the initial and final baryon states as specified in Table II. For simplicity and calculational advantages we mostly adopt a Gaussian form for the functions $\Phi_{B}$, i.e., we write

$$
\begin{aligned}
\Phi_{B}\left(\sum_{i<j}\left(x_{i}-x_{j}\right)^{2}\right) & =\int \frac{d q_{1}}{(2 \pi)^{4}} \int \frac{d q_{2}}{(2 \pi)^{4}} e^{-i q_{1}\left(x_{1}-x_{3}\right)-i q_{2}\left(x_{2}-x_{3}\right)} \tilde{\Phi}_{B}\left(-\vec{\Omega}^{2}\right), \\
\tilde{\Phi}_{B}\left(-\vec{\Omega}^{2}\right) & =\exp \left(\vec{\Omega}^{2} / \Lambda_{B}^{2}\right), \quad \vec{\Omega}^{2}=\frac{1}{2}\left(q_{1}+q_{2}\right)^{2}+\frac{1}{6}\left(q_{1}-q_{2}\right)^{2}=\frac{2}{3} \sum_{i \leq j} q_{i} q_{j},
\end{aligned}
$$

where $\Lambda_{B}$ is the size parameter for a given baryon with values of the order of $1 \mathrm{GeV}$. The size parameter represents the extension of the distribution of the constituent quarks in the given baryon.

\section{MATRIX ELEMENTS, HELICITY AMPLITUDES, AND RATE EXPRESSIONS}

The matrix element of the decay $\frac{1}{2}^{+} \rightarrow \frac{1}{2}^{+}+0^{-}$is written as

$$
M\left(B_{1} \rightarrow B_{2}+M\right)=M_{\mathrm{SD}}\left(B_{1} \rightarrow B_{2}+M\right)+M_{\mathrm{LD}_{1}}\left(B_{1} \rightarrow B_{\mathrm{res}} \rightarrow B_{2}+M\right)+M_{\mathrm{LD}_{2}}\left(B_{1} \rightarrow B_{\mathrm{res}}^{\prime}+M \rightarrow B_{2}+M\right) .
$$

Here

$$
\begin{gathered}
M_{\mathrm{SD}}=i^{4} \bar{u}\left(p_{2}\right) \Gamma_{B_{1} B_{2} M}\left(p_{1}, p_{2}, q\right) u\left(p_{1}\right), \\
\Gamma_{B_{1} B_{2} M}=g_{B_{1}} g_{B_{2}} g_{M} \int d x e^{-i p_{1} x} \int d y e^{i p_{2} y} \int d v e^{i q v} \int d z\left\langle T\left\{J_{B_{2}}(y) \mathcal{H}_{\mathrm{eff}}(z) J_{M}(v) \bar{J}_{B_{1}}(x)\right\}\right\rangle_{0}, \\
M_{\mathrm{LD}_{1}}=i^{6} \int \frac{d^{4} k}{(2 \pi)^{4} i} \bar{u}\left(p_{2}\right) \Gamma_{B_{\mathrm{res}} M B_{2}}\left(k, p_{2}, q\right) S_{B_{\mathrm{res}}}(k) \Gamma_{B_{1} B_{\mathrm{res}}}\left(p_{1}, k\right) u\left(p_{1}\right), \\
\Gamma_{B_{\mathrm{res}} M B_{2}}=g_{B_{\mathrm{res}}} g_{M} g_{B_{2}} \int d \xi_{2} e^{-i k \xi_{2}} \int d y e^{i p_{2} y} \int d v e^{i q v}\left\langle T\left\{J_{B_{2}}(y) J_{M}(v) \bar{J}_{B_{\mathrm{res}}}\left(\xi_{2}\right)\right\}\right\rangle_{0} \\
\Gamma_{B_{1} B_{\mathrm{res}}}=g_{B_{1}} g_{B_{\mathrm{res}}} \int d x e^{-i p_{1} x} \int d \xi_{1} e^{i k \xi_{1}} \int d z\left\langle T\left\{J_{B_{\mathrm{res}}}\left(\xi_{1}\right) \mathcal{H}_{\mathrm{eff}}(z) \bar{J}_{B_{1}}(x)\right\}\right\rangle_{0} \\
M_{\mathrm{LD}_{2}}=i^{6} \int \frac{d^{4} k}{(2 \pi)^{4} i} \bar{u}\left(p_{2}\right) \Gamma_{B_{\mathrm{res}} B_{2}}\left(k, p_{2}\right) S_{B_{\mathrm{res}}}(k) \Gamma_{B_{1} M B_{\mathrm{res}}}\left(p_{1}, k, q\right) u\left(p_{1}\right), \\
\Gamma_{B_{\mathrm{res}} B_{2}}=g_{B_{\mathrm{res}}} g_{B_{2}} \int d \xi_{2} e^{-i k \xi_{2}} \int d y e^{i p_{2} y} \int d z\left\langle T\left\{J_{B_{2}}(y) \mathcal{H}_{\mathrm{eff}}(z) \bar{J}_{B_{\mathrm{res}}}\left(\xi_{2}\right)\right\}\right\rangle_{0} \\
\Gamma_{B_{1} M B_{\mathrm{res}}}=g_{B_{1}} g_{M} g_{B_{\mathrm{res}}} \int d x e^{-i p_{1} x} \int d v e^{i q v} \int d \xi_{1} e^{i k \xi_{1}}\left\langle T\left\{J_{B_{\mathrm{res}}}\left(\xi_{1}\right) J_{M}(v) \bar{J}_{B_{1}}(x)\right\}\right\rangle_{0}
\end{gathered}
$$


In our approach the building blocks $\Gamma$ are represented by a set of the Feynman diagrams shown in Fig. 3 for the SD contributions and in Fig. 4 for the LD contributions.

By going to the momentum space in the above building blocks, one gets

$$
\begin{aligned}
\Gamma_{B_{1} B_{2} M}\left(p_{1}, p_{2}, q\right) & =i(2 \pi)^{4} \delta\left(p_{1}-p_{2}-q\right) \frac{G_{F}}{\sqrt{2}} V_{\mathrm{CKM}} \tilde{\Gamma}_{B_{1} B_{2} M}\left(p_{1}, p_{2}\right), \\
\Gamma_{B_{1} B_{\mathrm{res}}}\left(p_{1}, k\right) & =i(2 \pi)^{4} \delta\left(p_{1}-k\right) \frac{G_{F}}{\sqrt{2}} V_{\mathrm{CKM}} \tilde{\Gamma}_{B_{1} B_{\mathrm{res}}}\left(p_{1}\right), \\
\Gamma_{B_{\mathrm{res}} M B_{2}}\left(k, p_{2}, q\right) & =i(2 \pi)^{4} \delta\left(k-p_{2}-q\right) \frac{G_{F}}{\sqrt{2}} V_{\mathrm{CKM}} \tilde{\Gamma}_{B_{\mathrm{res}} M B_{2}}\left(k, p_{2}\right), \\
\Gamma_{B_{\mathrm{res}} B_{2}}\left(k, p_{2}\right) & =i(2 \pi)^{4} \delta\left(k-p_{2}\right) \frac{G_{F}}{\sqrt{2}} V_{\mathrm{CKM}} \tilde{\Gamma}_{B_{\mathrm{res}} B_{2}}\left(p_{2}\right), \\
\Gamma_{B_{1} M B_{\mathrm{res}}}\left(p_{1}, k, q\right) & =i(2 \pi)^{4} \delta\left(p_{1}-k-q\right) \frac{G_{F}}{\sqrt{2}} V_{\mathrm{CKM}} \tilde{\Gamma}_{B_{1} M B_{\mathrm{res}}}\left(p_{1}, k\right),
\end{aligned}
$$

where $V_{\mathrm{CKM}}=V_{\mathrm{ud}} V_{\mathrm{us}}^{*}$. The Wilson coefficients $C_{1}$ and $C_{2}$ appear in the combinations $C_{2}+\xi C_{1}$ for charged mesons and $C_{1}+\xi C_{2}$ for neutral mesons $\left(\xi=1 / N_{c}\right)$ in the case of the tree diagrams Ia and Ib. In the case of other diagrams including the pole diagrams they appear in the combinations $C_{2}-C_{1}$. The details of calculation of two- and threeloop quark diagrams in CCQM may be found in our previous papers (see Refs. [4-14]).

Let us discuss some subtleties of calculation of the pole diagrams. There are contributions from the $\frac{1}{2}^{+}$resonances (neutron and $\left.\Sigma^{+}\right)$. Note that the $\frac{3}{2}^{+}$resonances $\left(\Delta^{0}\right.$ and $\Sigma^{*+}$ ) do not contribute to the amplitude due to the KPW theorem $[59,60]$. This theorem states that the contraction of the flavor-antisymmetric current-current operator with a flavorsymmetric final state configuration is zero in the SU(3) limit. The antisymmetric [us] diquark emerging from the weak vertex is in the $3^{*}$ representation and cannot evolve

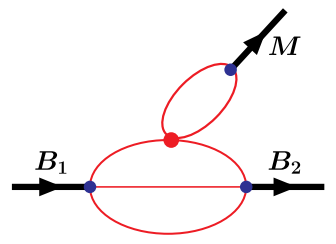

tree diagrams $\mathrm{Ia}, \mathrm{Ib}$

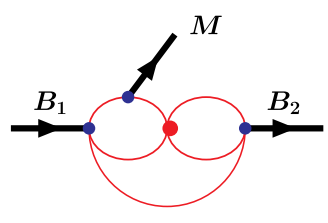

W-exchange diagram IIb

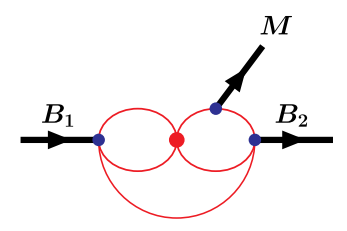

W-exchange diagram IIa

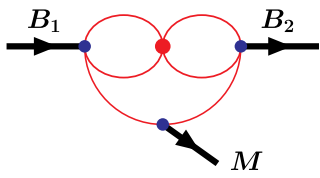

W-exchange diagram III
FIG. 3. Feynman diagrams describing the SD contributions. into the 6 representation of the symmetric final-state $\{$ us $\}$ diquark.

The propagator of the $\frac{1}{2}^{+}$resonances is the ordinary Dirac propagator,

$$
S(p)=\frac{1}{m_{\mathrm{res}}-\not p}=\frac{m_{\mathrm{res}}+\not p}{m_{\mathrm{res}}^{2}-p^{2}} .
$$

Let us consider in detail the calculation of the $\Lambda \rightarrow$ $p+\pi^{-}$process which goes via neutron and $\Sigma^{+}$resonances. Recalling Eqs. (7) and (8) one has

$$
M_{\mathrm{LD}_{1 ; 2}}=i(2 \pi)^{4} \delta\left(p_{1}-p_{2}-q\right) \frac{G_{F}}{\sqrt{2}} V_{\mathrm{CKM}} \tilde{M}_{\mathrm{LD}_{1 ; 2}}
$$

where

$$
\begin{aligned}
& \tilde{M}_{\mathrm{LD}_{1}}=-\bar{u}\left(p_{2}\right) \tilde{\Gamma}_{n \pi p}\left(p_{1}, p_{2}\right) S_{n}\left(p_{1}\right) \tilde{\Gamma}_{\Lambda n}\left(p_{1}\right) u\left(p_{1}\right), \\
& \tilde{M}_{\mathrm{LD}_{2}}=-\bar{u}\left(p_{2}\right) \tilde{\Gamma}_{\Sigma^{+} p}\left(p_{2}\right) S_{\Sigma^{+}}\left(p_{2}\right) \tilde{\Gamma}_{\Lambda \pi \Sigma^{+}}\left(p_{1}, p_{2}\right) u\left(p_{1}\right) .
\end{aligned}
$$

By using the Dirac equations of motion $\bar{u}\left(p_{2}\right) \not p_{2}=$ $m_{2} \bar{u}\left(p_{2}\right)$ and $\not p_{1} u\left(p_{1}\right)=m_{1} u\left(p_{1}\right)$ one can get

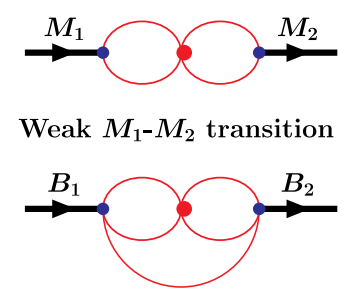

Weak $B_{1}-B_{2}$ transition

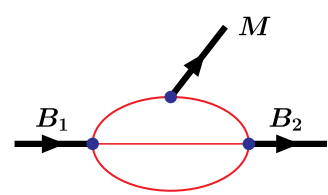

Strong $B_{1} B_{2} M$ coupling
FIG. 4. Feynman diagrams describing the building blocks of pole contributions. 


$$
\begin{aligned}
\bar{u}\left(p_{2}\right) \tilde{\Gamma}_{n \pi p}\left(p_{1}, p_{2}\right) & =\bar{u}\left(p_{2}\right) \gamma_{5}\left(C_{n \pi p}+\not \phi_{1} D_{n \pi p}\right), & \tilde{\Gamma}_{\Lambda n}\left(p_{1}\right) u\left(p_{1}\right)=\left(A_{\Lambda n}+\gamma_{5} B_{\Lambda n}\right) u\left(p_{1}\right), \\
\bar{u}\left(p_{2}\right) \tilde{\Gamma}_{\Sigma^{+} p}\left(p_{2}\right) & =\bar{u}\left(p_{2}\right)\left(A_{\Sigma^{+} p}+\gamma_{5} B_{\Sigma^{+} p}\right), & \tilde{\Gamma}_{\Lambda \pi \Sigma^{+}}\left(p_{1}, p_{2}\right) u\left(p_{1}\right)=\gamma_{5}\left(C_{\Lambda \pi \Sigma^{+}}+\not p_{1} D_{\Lambda \pi \Sigma^{+}}\right) .
\end{aligned}
$$

Finally, we arrive at the invariant matrix elements for the pole diagrams with intermediate $\frac{1}{2}^{+}$resonances. One has

$$
\begin{aligned}
\tilde{M}_{\mathrm{LD}_{1}} & \equiv \tilde{M}_{n}=\bar{u}\left(p_{2}\right)\left(A_{n}+\gamma_{5} B_{n}\right) u\left(p_{1}\right), \\
A_{n} & =-\frac{B_{\Lambda n}\left(C_{n \pi p}-m_{\Lambda} D_{n \pi p}\right)}{m_{n}+m_{\Lambda}}, \quad B_{n}=-\frac{A_{\Lambda n}\left(C_{n \pi p}+m_{\Lambda} D_{n \pi p}\right)}{m_{n}-m_{\Lambda}}, \\
\tilde{M}_{\mathrm{LD}_{2}} & \equiv \tilde{M}_{\Sigma}=\bar{u}\left(p_{2}\right)\left(A_{\Sigma}+\gamma_{5} B_{\Sigma}\right) u\left(p_{1}\right), \\
A_{\Sigma} & =-\frac{B_{\Sigma^{+} p}\left(C_{\Lambda \pi \Sigma^{+}}-m_{p} D_{\Lambda \pi \Sigma^{+}}\right)}{m_{\Sigma}+m_{p}}, \quad B_{\Sigma}=-\frac{A_{\Sigma^{+} p}\left(C_{\Lambda \pi \Sigma^{+}}+m_{p} D_{\Lambda \pi \Sigma^{+}}\right)}{m_{\Sigma}-m_{p}} .
\end{aligned}
$$

The calculation of the $A$ and $B$ amplitudes appearing in the decay $\Lambda \rightarrow n+\pi^{0}$ proceeds in an analogous manner. The pole diagram with the kaon resonance contributes only to the structure which is proportional to $\gamma_{5}$. Numerically, they are negligibly small.

It is well known that the $P$-wave amplitude $B$ is dominated by the low-lying $1 / 2^{+}$resonances whereas their contributions are tiny to the $S$-wave amplitude $A$. The invariant amplitudes $A$ and $B$ may be converted to a set of helicity amplitudes $H_{\lambda_{1} \lambda_{M}}$ as described in [58]. One has

$$
H_{\frac{1}{2} t}^{V}=\sqrt{Q_{+}} A, \quad H_{\frac{1}{2} t}^{A}=\sqrt{Q_{-}} B
$$

where $m_{ \pm}=m_{1} \pm m_{2}, Q_{ \pm}=m_{ \pm}^{2}-q^{2}$.

We show in Fig. 5 the behavior of the helicity amplitudes as a function of the size parameter $\Lambda_{s}$. One can clearly see that the $S$-wave amplitudes are almost zero.

It is widely accepted that $S$-wave amplitude is saturated by the $\frac{1}{2}^{-}$resonances, see, e.g., Refs. [61-66]. Ordinarily, their contributions are calculated by using the well-known

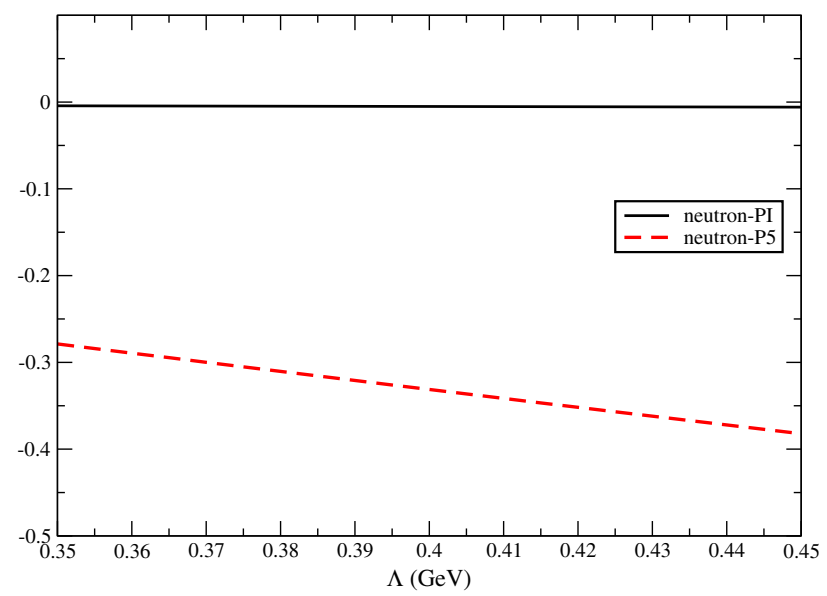

soft-pion theorem in the current-algebra approach. It allows one to express the parity-violating $S$-wave amplitude in terms of parity-conserving matrix elements. In our case, one has, see, e.g., [66]

$$
\begin{aligned}
& A_{1 / 2^{-}}\left(\Lambda \rightarrow p+\pi^{-}\right)=\frac{1}{f_{\pi}} A_{\Lambda n}, \\
& A_{1 / 2^{-}}\left(\Lambda \rightarrow n+\pi^{0}\right)=\frac{1}{\sqrt{2} f_{\pi}} A_{\Lambda n},
\end{aligned}
$$

where $f_{\pi}$ is the leptonic pion decay constant. Here we adopt our convention of signs.

Finally, the transition $\frac{1}{2}^{+} \rightarrow \frac{1}{2}^{+}+0^{-}$amplitude is written in terms of invariant amplitudes as

$$
\left\langle B_{2} P\left|\mathcal{H}_{\mathrm{eff}}\right| B_{1}\right\rangle=\frac{G_{F}}{\sqrt{2}} V_{\mathrm{CKM}} \bar{u}\left(p_{2}\right)\left(A+\gamma_{5} B\right) u\left(p_{1}\right),
$$

where $A$ and $B$ include all relevant contributions discussed above.

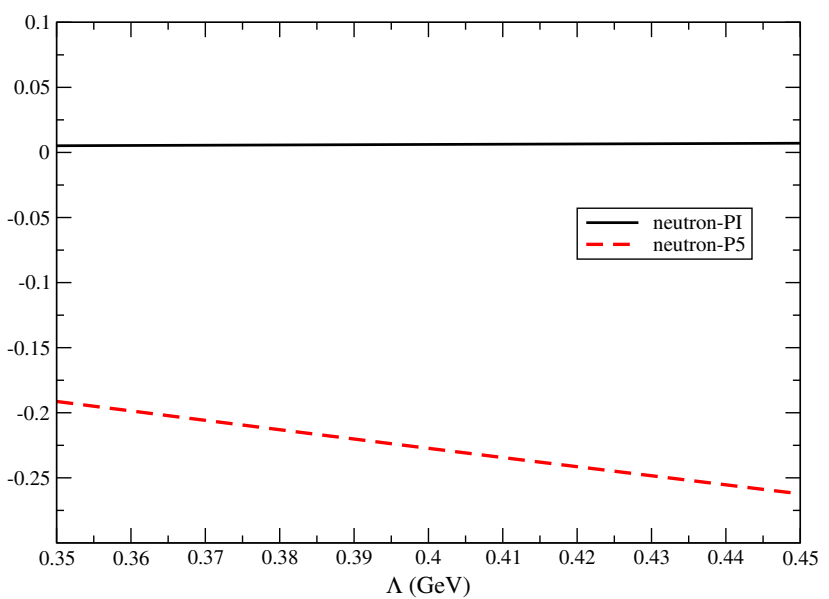

FIG. 5. Dependence of the helicities $P I \equiv H_{1 / 2 t}^{V}$ and $P 5 \equiv H_{1 / 2 t}^{A}$ on the size parameter in the case of the neutron resonance. Left panel: the decay $\Lambda \rightarrow p+\pi$; right panel: the decay $\Lambda \rightarrow n+\pi$. 
The two-body decay widths read

$$
\begin{aligned}
\Gamma\left(B_{1} \rightarrow B_{2}+P\right) & =\frac{G_{F}^{2}}{32 \pi} \frac{\left|\mathbf{p}_{2}\right|}{m_{1}^{2}}\left|V_{\mathrm{CKM}}\right|^{2} \mathcal{H}_{S}, \\
\mathcal{H}_{S} & =2\left(\left|H_{\frac{1}{2} t}^{V}\right|^{2}+\left|H_{\frac{1}{2} t}^{A}\right|^{2}\right),
\end{aligned}
$$

where $\left|\mathbf{p}_{\mathbf{2}}\right|=\lambda^{1 / 2}\left(m_{1}^{2}, m_{2}^{2}, q^{2}\right) /\left(2 m_{1}\right)$.

\section{NUMERICAL RESULTS}

Our covariant constituent quark model contains a number of model parameters which have been determined by a global fit to a multitude of decay processes. The values of the constituent quark masses $m_{q}$ are taken from the last fit in [67]. In the fit, the infrared cutoff parameter $\lambda$ of the model has been kept fixed as found in the original paper [68]. One has

$$
\begin{array}{cccccc}
m_{u} & m_{s} & m_{c} & m_{b} & \lambda & \\
\hline 0.242 & 0.428 & 1.672 & 5.046 & 0.181 & \mathrm{GeV}
\end{array}
$$

The size parameters of light mesons were fixed by fitting the data on the leptonic decay constants. The numerical values of the size parameters and the leptonic decay constants for pion and kaon are shown in Eq. (20).

\begin{tabular}{cccc} 
Meson & $\Lambda_{M}(\mathrm{GeV})$ & $f_{M}(\mathrm{MeV})$ & $f_{M}^{\text {expt }}(\mathrm{MeV})$ \\
\hline Pion & 0.871 & 130.3 & $130.0 \pm 1.7$ \\
Kaon & 1.014 & 156.0 & $156.1 \pm 0.8$
\end{tabular}

In case of the nucleons, the best description of magnetic moments, electromagnetic radii, and form factors is achieved in [69] for a superposition of the $V$ and $T$ currents of nucleons according to Table II with $x=0.8$ and $\Lambda_{N}=0.5 \mathrm{GeV}$. The $\Lambda$ size parameter is the only adjustable parameter. In Fig. 6 we plot the dependence on this parameter of two branching rates $\Lambda \rightarrow p+\pi^{-}$and $\Lambda \rightarrow n+\pi^{0}$. One can see that the theoretical curves fit the data for $\Lambda \approx 0.355 \mathrm{GeV}$ in both decays simultaneously. The given value of $0.355 \mathrm{GeV}$ differs from the value of $0.492 \mathrm{GeV}$ fixed in our previous paper [70] by fitting the experimental data on the magnetic moment of the $\Lambda$ hyperon. The point is that the calculated branching fractions depend very strongly on the size parameter $\Lambda$ as one can see from Fig. 6 . Contrary, the magnetic moment of the $\Lambda$ hyperon calculated in the indicated paper depends very weakly on this parameter. We have taken our old FORTRAN code and recalculated its value for $0.355 \mathrm{GeV}$. We found that $\mu_{\Lambda}=$ -0.74 for $0.355 \mathrm{GeV}$, which is very close to the old result, $\mu_{\Lambda}=-0.73$ for $0.492 \mathrm{GeV}$.

For comparison, we plot the SD contributions coming from the diagrams with topologies Ia (charged pion), Ib
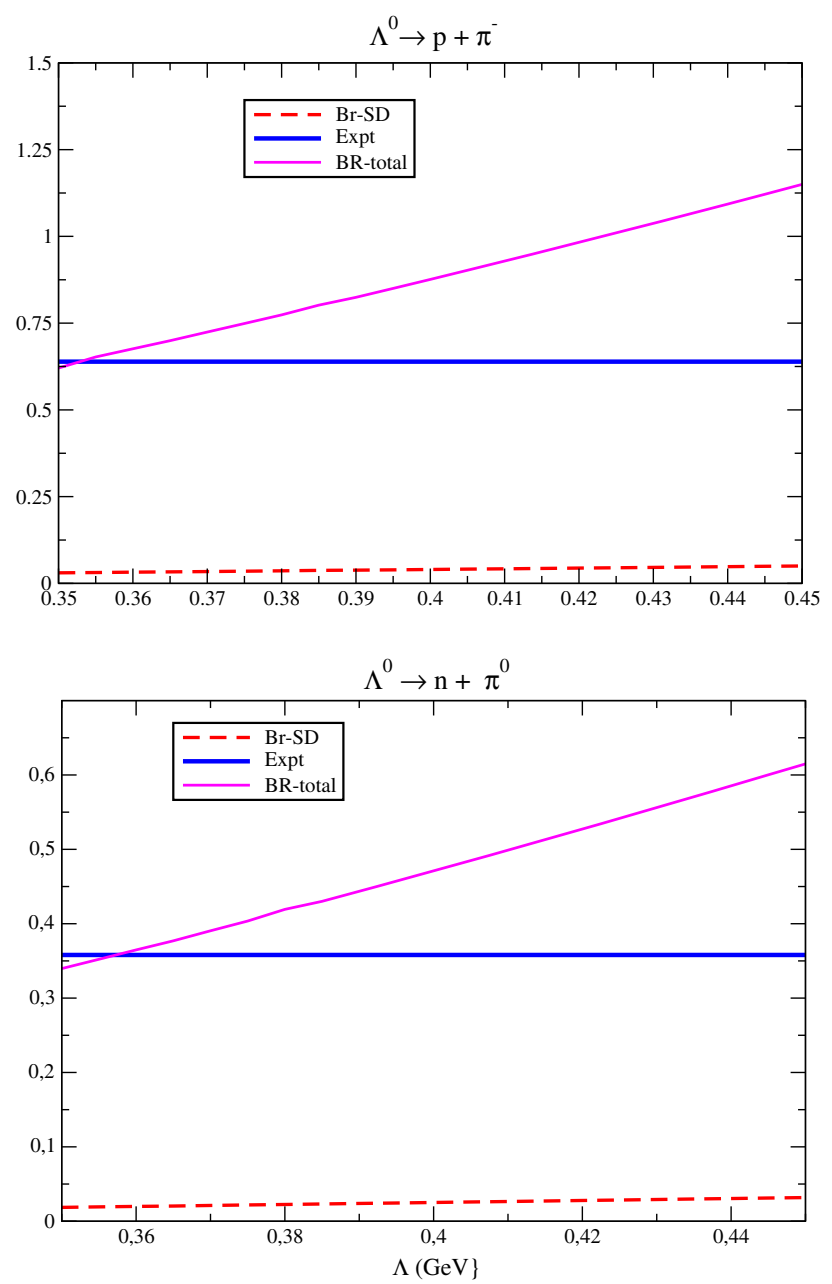

FIG. 6. Dependence of the nonleptonic $\Lambda$ decay widths on its size parameter.

(neutral pion), and IIa, IIb, and III (all two modes). It is readily seen that their contributions are relatively less than those coming from the pole diagrams. However, the calculation of those diagrams is time-consuming because it involves the analytical and numerical calculation of three loops. The most significant contributions among the pole diagrams are coming from the diagrams with intermediate neutron resonance with mass closest to the $\Lambda$ resonance. The contribution from the pole diagram with intermediate kaon resonance is negligibly small.

In Tables III-VI the numerical results of $A_{\mathrm{SD}}, A_{\mathrm{LD}}$ and $B_{\mathrm{SD}}, B_{\mathrm{LD}}$ at $\Lambda=0.355 \mathrm{GeV}$ are shown. One can see that the $B$ amplitudes dominate over $A$ amplitudes. The SD contributions are suppressed almost by one order of magnitude comparison with the LD contributions. The numerical results for the full amplitudes are written down.

$$
\begin{array}{ll}
\boldsymbol{\Lambda} \rightarrow \mathbf{p} \pi^{-}: A=A_{\mathrm{SD}}+A_{\mathrm{LD}}=+0.124 \mathrm{GeV}^{2}, & B=B_{\mathrm{SD}}+B_{\mathrm{LD}}=-3.042 \mathrm{GeV}^{2} . \\
\Lambda \rightarrow \mathbf{n} \pi^{\mathbf{0}}: A=A_{\mathrm{SD}}+A_{\mathrm{LD}}=+0.087 \mathrm{GeV}^{2}, & B=B_{\mathrm{SD}}+B_{\mathrm{LD}}=-2.059 \mathrm{GeV}^{2} .
\end{array}
$$


TABLE III. SD contributions to the amplitudes $A$ and $B$ of the decay $\Lambda \rightarrow p \pi^{-}$in units of $\mathrm{GeV}^{2}$.

\begin{tabular}{lccccc}
\hline \hline Amplitudes & Ia & IIa & IIb & III & Sum (SD) \\
\hline$A_{\mathrm{SD}}$ & $-0.372 \times 10^{-1}$ & $0.269 \times 10^{-3}$ & $0.300 \times 10^{-1}$ & $0.213 \times 10^{-1}$ & $0.144 \times 10^{-1}$ \\
$B_{\mathrm{SD}}$ & -0.345 & -0.116 & 0.167 & -0.452 & -0.746 \\
\hline \hline
\end{tabular}

TABLE IV. LD contributions to the amplitudes $A$ and $B$ of the decay $\Lambda \rightarrow p \pi^{-}$in units of $\mathrm{GeV}^{2}$.

\begin{tabular}{|c|c|c|c|c|c|c|}
\hline Amplitudes & $n$ & $\Sigma^{+}$ & $K$ & $K^{*}$ & $\frac{1}{2}^{-}$(Soft pion) & Sum (LD) \\
\hline$A_{\mathrm{LD}}$ & $-2.13 \times 10^{-3}$ & $-9.54 \times 10^{-3}$ & 0 & $2.61 \times 10^{-2}$ & $0.869 \times 10^{-1}$ & $1.10 \times 10^{-1}$ \\
\hline$B_{\mathrm{LD}}$ & -2.55 & $2.26 \times 10^{-1}$ & $2.82 \times 10^{-2}$ & 0 & 0 & -2.296 \\
\hline
\end{tabular}

TABLE V. SD contributions to the amplitudes $A$ and $B$ of the decay $\Lambda \rightarrow n \pi^{0}$ in units of $\mathrm{GeV}^{2}$.

\begin{tabular}{lccccc}
\hline \hline Amplitudes & Ib & IIa & IIb & III & Sum (SD) \\
\hline$A_{\mathrm{SD}}$ & $-0.120 \times 10^{-1}$ & $0.190 \times 10^{-3}$ & $0.211 \times 10^{-1}$ & $0.150 \times 10^{-1}$ & $0.243 \times 10^{-1}$ \\
$B_{\mathrm{SD}}$ & -0.112 & $-0.82 \times 10^{-1}$ & 0.119 & -0.319 & -0.394 \\
\hline \hline
\end{tabular}

TABLE VI. LD contributions to the amplitudes $A$ and $B$ of the decay $\Lambda \rightarrow n \pi^{0}$ in units of $\mathrm{GeV}^{2}$.

\begin{tabular}{lcccccc}
\hline \hline Amplitudes & $n$ & $\Sigma^{0}$ & $K$ & $K^{*}$ & $\frac{1}{2}$ (Soft pion) & Sum (LD) \\
\hline$A_{\mathrm{LD}}$ & $-1.52 \times 10^{-3}$ & $-6.58 \times 10^{-3}$ & 0 & $8.44 \times 10^{-3}$ & $6.24 \times 10^{-2}$ & $0.627 \times 10^{-1}$ \\
$B_{\mathrm{LD}}$ & -1.83 & $1.56 \times 10^{-1}$ & $0.902 \times 10^{-2}$ & 0 & 0 & -1.665 \\
\hline \hline
\end{tabular}

\section{SUMMARY AND CONCLUSION}

We have studied two-body nonleptonic decays of light lambda hyperon $\Lambda \rightarrow p \pi^{-}\left(n \pi^{0}\right)$ with account for both short- and long-distance effects. The short-distance effects are induced by five topologies of external and internal weak $W$ interactions, while long-distance effects are saturated by an inclusion of the so-called pole diagrams. Pole diagrams are generated by resonance contributions of the low-lying spin $\frac{1}{2}^{+}$(nucleon and $\Sigma$ ) and spin $\frac{1}{2}-$ baryons. The last contributions are calculated by using the well-known softpion theorem. The spin $\frac{3}{2}+$ resonances do not contribute to the amplitude due to the Körner-Pati-Woo theorem. The contributions from the intermediate $K$ meson is also negligibly small. From our previous analysis of heavy baryons we have known that short-distance effects induced by internal topologies are not suppressed in comparison with external $W$-exchange diagrams and must be included for description of data. Here, in the case of $\Lambda$ decays we have found that the contribution of the SD diagrams is sizably suppressed, almost by one order of magnitude in comparison with data, which are known with quite good accuracy. The most significant contributions are coming from the intermediate $\frac{1}{2}^{+}$and $\frac{1}{2}^{-}$resonances. The contribution from the kaon resonance is negligibly small.

\section{ACKNOWLEDGMENTS}

This work was funded by BMBF "Verbundprojekt No. 05P2018-Ausbau von ALICE am LHC: Jets und partonische Struktur von Kernen" (Förderkennzeichen No. 05P18VTCA1), by ANID PIA/APOYO No. AFB180002, by FONDECYT (Chile) under Grant No. 1191103, and by Millennium Institute for Subatomic Physics at the High-Energy Frontier (SAPHIR) of ANID, Code No. ICN2019_044 (Chile). M. A. I. acknowledges the support from the PRISMA Cluster of Excellence (Mainz Uni.). M. A. I. and J.G. K. acknowledge the Heisenberg-Landau Grant for partial support. Z.T.'s research has been funded by the Science Committee of the Ministry of Education and Science of the Republic of Kazakhstan (Grant No. AP09057862). 
[1] P. A. Zyla et al. (Particle Data Group), Prog. Theor. Exp. Phys. 2020, 083C01 (2020).

[2] A. K. Leibovich, Z. Ligeti, I. W. Stewart, and M. B. Wise, Phys. Lett. B 586, 337 (2004).

[3] G. Buchalla, A. J. Buras, and M. E. Lautenbacher, Rev. Mod. Phys. 68, 1125 (1996).

[4] M. A. Ivanov, J. G. Körner, and V. E. Lyubovitskij, Fiz. Elem. Chastits At. Yadra 51, 678 (2020) [Phys. Part. Nucl. 51, 678 (2020)].

[5] M. A. Ivanov, Particles 3, 23 (2020).

[6] T. Gutsche, M. A. Ivanov, J. G. Körner, V. E. Lyubovitskij, and Z. Tyulemissov, Phys. Rev. D 100, 114037 (2019).

[7] T. Gutsche, M. A. Ivanov, J. G. Körner, and V. E. Lyubovitskij, Particles 2, 339 (2019).

[8] T. Gutsche, M. A. Ivanov, J. G. Körner, V. E. Lyubovitskij, and Z. Tyulemissov, Phys. Rev. D 99, 056013 (2019).

[9] T. Gutsche, M. A. Ivanov, J. G. Körner, and V. E. Lyubovitskij, Phys. Rev. D 98, 074011 (2018).

[10] T. Gutsche, M. A. Ivanov, J. G. Körner, and V. E. Lyubovitskij, Phys. Rev. D 96, 054013 (2017).

[11] T. Gutsche, M. A. Ivanov, J. G. Körner, V. E. Lyubovitskij, V. V. Lyubushkin, and P. Santorelli, Phys. Rev. D 96, 013003 (2017).

[12] T. Gutsche, M. A. Ivanov, J. G. Körner, V. E. Lyubovitskij, and P. Santorelli, Phys. Rev. D 92, 114008 (2015).

[13] M. A. Ivanov, J. G. Körner, V. E. Lyubovitskij, and A. G. Rusetsky, Phys. Rev. D 57, 5632 (1998).

[14] M. A. Ivanov, J. G. Körner, V. E. Lyubovitskij, and A. G. Rusetsky, Mod. Phys. Lett. A 13, 181 (1998).

[15] J. J. Sakurai, Phys. Rev. 156, 1508 (1967).

[16] A. P. Balachandran, M. G. Gundzik, and S. Pakvasa, Phys. Rev. 153, 1553 (1967).

[17] S. Nussinov and G. Preparata, Phys. Rev. 175, 2180 (1968).

[18] F. C. P. Chan, Phys. Rev. 171, 1543 (1968); Phys. Rev. D 7 , 287(E) (1973).

[19] C. H. Albright, Phys. Rev. 187, 1880 (1969).

[20] A. Hosoya and N. Tokuda, Lett. Nuovo Cimento 1, 235 (1971).

[21] Y. E. Nagai, Phys. Rev. D 5, 60 (1972).

[22] M. D. Scadron and L. R. Thebaud, Phys. Rev. D 8, 2190 (1973).

[23] K. J. Sebastian, Phys. Rev. D 8, 2296 (1973).

[24] M. P. Khanna, Phys. Rev. D 13, 1266 (1976).

[25] M. Nakagawa and N. N. Trofimenkoff, Nucl. Phys. B5, 93 (1968).

[26] M. A. Shifman, A. I. Vainshtein, and V. I. Zakharov, Zh. Eksp. Teor. Fiz. 72, 1275 (1977) [Sov. Phys. JETP 45, 670 (1977)].

[27] B. W. Lee, Phys. Rev. 170, 1359 (1968).

[28] J. Bijnens, H. Sonoda, and M. B. Wise, Nucl. Phys. B261, 185 (1985).

[29] E. E. Jenkins, Nucl. Phys. B375, 561 (1992).

[30] C. Carone and H. Georgi, Nucl. Phys. B375, 243 (1992).

[31] B. Borasoy and B. R. Holstein, Phys. Rev. D 59, 094025 (1999).

[32] B. Borasoy and B. R. Holstein, Eur. Phys. J. C 6, 85 (1999).

[33] R. P. Springer, Phys. Lett. B 461, 167 (1999).

[34] A. Abd El-Hady and J. Tandean, Phys. Rev. D 61, 114014 (2000).

[35] R. Flores-Mendieta, Phys. Rev. D 99, 094033 (2019).
[36] J. Tandean, Phys. Rev. D 69, 076008 (2004).

[37] G. Nardulli, Nuovo Cimento A 100, 485 (1988).

[38] D. D. Wu and J. L. Rosner, Phys. Rev. D 33, 1367 (1986).

[39] J. F. Donoghue, E. Golowich, and Y. C. R. Lin, Phys. Rev. D 32, 1733 (1985); 33, 2728(E) (1986).

[40] M. Praszalowicz and J. Trampetic, Fizika 18, 391 (1986).

[41] H. Galic, D. Tadic, and J. Trampetic, Nucl. Phys. B158, 306 (1979).

[42] V. N. Pervushin and N. A. Sarikov, Yad. Fiz. 41, 1361 (1985) [Sov. J. Nucl. Phys. 41, 865 (1985)].

[43] K. Terasaki, Lett. Nuovo Cimento 18, 376 (1977).

[44] B. Guberina and D. Tadic, Phys. Rev. D 18, 2522 (1978).

[45] M. Katuya, Phys. Lett. 83B, 227 (1979).

[46] C. Schmid, Phys. Lett. 66B, 353 (1977).

[47] A. Le Yaouanc, L. Oliver, O. Pene, and J.-C. Raynal, Phys. Lett. 72B, 53 (1977).

[48] T. Hayashi, T. Karino, and T. Yanagida, Prog. Theor. Phys. 60, 1066 (1978).

[49] Y. Abe, M. Shin-Mura, and T. Uchida, Prog. Theor. Phys. 77, 1023 (1987).

[50] Y. G. Xu, X. D. Cheng, J. L. Zhang, and R. M. Wang, J. Phys. G 47, 085005 (2020).

[51] R. M. Wang, M. Z. Yang, H. B. Li, and X. D. Cheng, Phys. Rev. D 100, 076008 (2019).

[52] A. Y. Berdnikov, Y. A. Berdnikov, V. A. Ivanova, A. V. Nikitchenko, N. I. Troitskaya, M. Faber, and A. N. Ivanov, Int. J. Mod. Phys. A 22, 1835 (2007).

[53] P. Zenczykowski, Phys. Rev. D 73, 076005 (2006).

[54] M. Cristoforetti, P. Faccioli, E. V. Shuryak, and M. Traini, Phys. Rev. D 70, 054016 (2004).

[55] E. Hiyama, K. Suzuki, H. Toki, and M. Kamimura, Prog. Theor. Phys. 112, 99 (2004).

[56] A. Garcia, R. Huerta, and G. Sanchez-Colon, J. Phys. G 25, 45 (1999).

[57] L. B. Okun, Leptons and Quarks (North Holland, Amsterdam, 1985), p. 362.

[58] J. G. Körner and M. Krämer, Z. Phys. C 55, 659 (1992).

[59] J. G. Körner, Nucl. Phys. B25, 282 (1971).

[60] J. C. Pati and C. H. Woo, Phys. Rev. D 3, 2920 (1971).

[61] R. E. Marshak, Riazuddin, and C. P. Ryan, Theory of Weak Interactions in Particle Physics (Wiley-Interscience, New York, 1969), p. 761.

[62] D. Bailin, Weak Interactions (Sussex University Press, Chatto \& Windus, 1977), p. 406.

[63] D. Ebert and W. Kallies, Yad. Fiz. 40, 1250 (1984) [Sov. J. Nucl. Phys. 40, 794 (1984)].

[64] D. Ebert and W. Kallies, Phys. Lett. B 131, 183 (1983); 148, 502(E) (1984).

[65] H. Y. Cheng, Z. Phys. C 29, 453 (1985).

[66] H. Y. Cheng, X. W. Kang, and F. Xu, Phys. Rev. D 97, 074028 (2018).

[67] T. Gutsche, M. A. Ivanov, J. G. Körner, V. E. Lyubovitskij, P. Santorelli, and N. Habyl, Phys. Rev. D 91, 074001 (2015); 91, 119907(E) (2015).

[68] T. Branz, A. Faessler, T. Gutsche, M. A. Ivanov, J. G. Körner, and V. E. Lyubovitskij, Phys. Rev. D 81, 034010 (2010).

[69] T. Gutsche, M. A. Ivanov, J. G. Körner, V. E. Lyubovitskij, and P. Santorelli, Phys. Rev. D 86, 074013 (2012).

[70] T. Gutsche, M. A. Ivanov, J. G. Körner, V. E. Lyubovitskij, and P. Santorelli, Phys. Rev. D 87, 074031 (2013). 